\title{
Re-education of macrophages as a therapeutic strategy in cancer
}

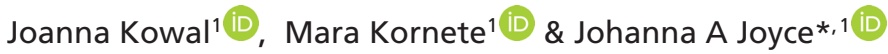 \\ ${ }^{1}$ Department of Oncology, Ludwig Institute for Cancer Research, University of Lausanne, Switzerland \\ *Author for correspondence: johanna@joycelab.org
}

Tumor-associated macrophages (TAMs) can be educated within the tumor microenvironment to promote cancer development and progression. While TAM-targeted agents have largely focused on macrophage depletion as an anticancer strategy, it is becoming increasingly evident that TAM re-education may represent a more effective approach. In this perspective, we discuss different means to achieve TAM reeducation, and review the beneficial effects of these strategies, particularly when combined with immune checkpoint inhibitors.

\section{Graphical abstract:}

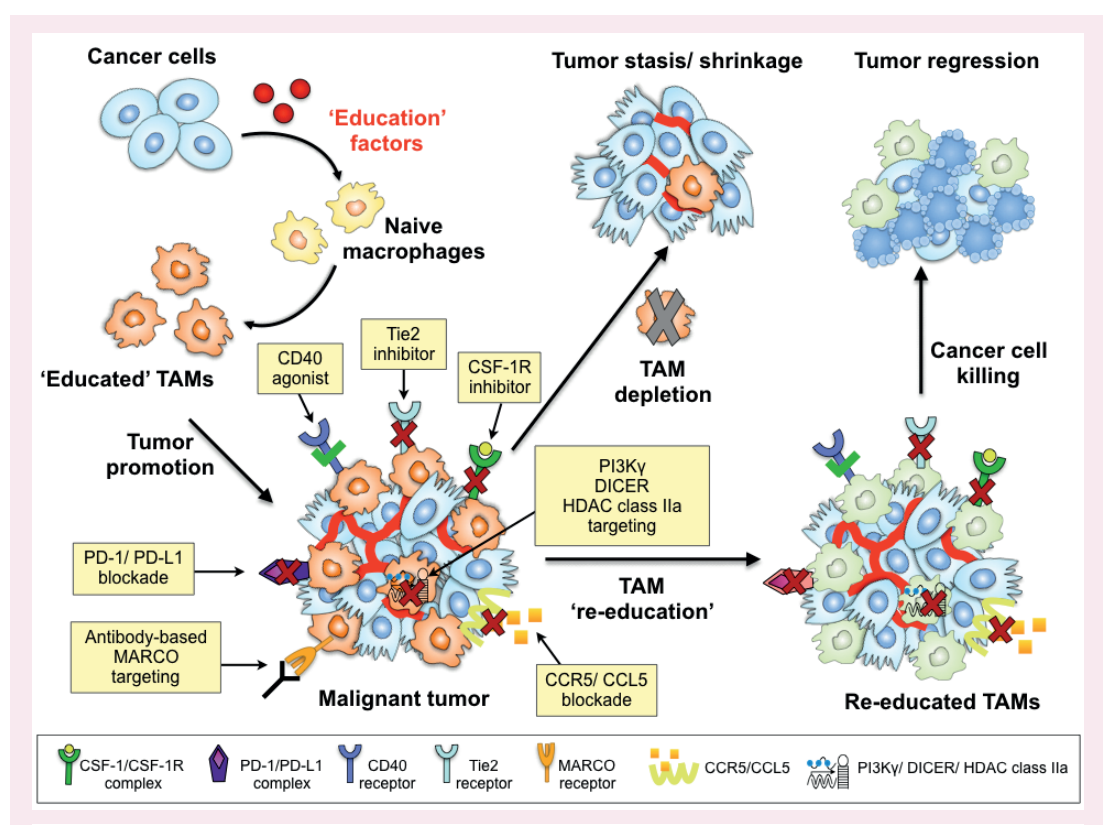

First draft submitted: 5 October 2018; Accepted for publication: 19 March 2018; Published online: 15 May 2019

Keywords: anticancer treatment $\bullet$ cancer immunotherapy $\bullet$ macrophages $\bullet$ tumor-associated macrophages $\bullet$ tumor microenvironment

\section{Tumor-associated macrophages in the cancer microenvironment}

Tumors develop and progress within a complex tissue environment involving a multitude of intercellular interactions, not only between heterogeneous cancer cells, but also with the many normal cell types that collectively comprise the tumor microenvironment (TME). The TME can be defined as the numerous innate and adaptive immune cell types, stromal cells, extracellular matrix, blood and lymphatic vessel networks that comprise the tumor mass along with the cancer cells. Normal cells in the TME are educated and can consequently be co-opted during the process of tumor progression to actively promote malignancy [1-3]. Although the precise composition of the TME varies in an organ-dependent manner, tumor-associated macrophages (TAMs) have emerged as one of the 


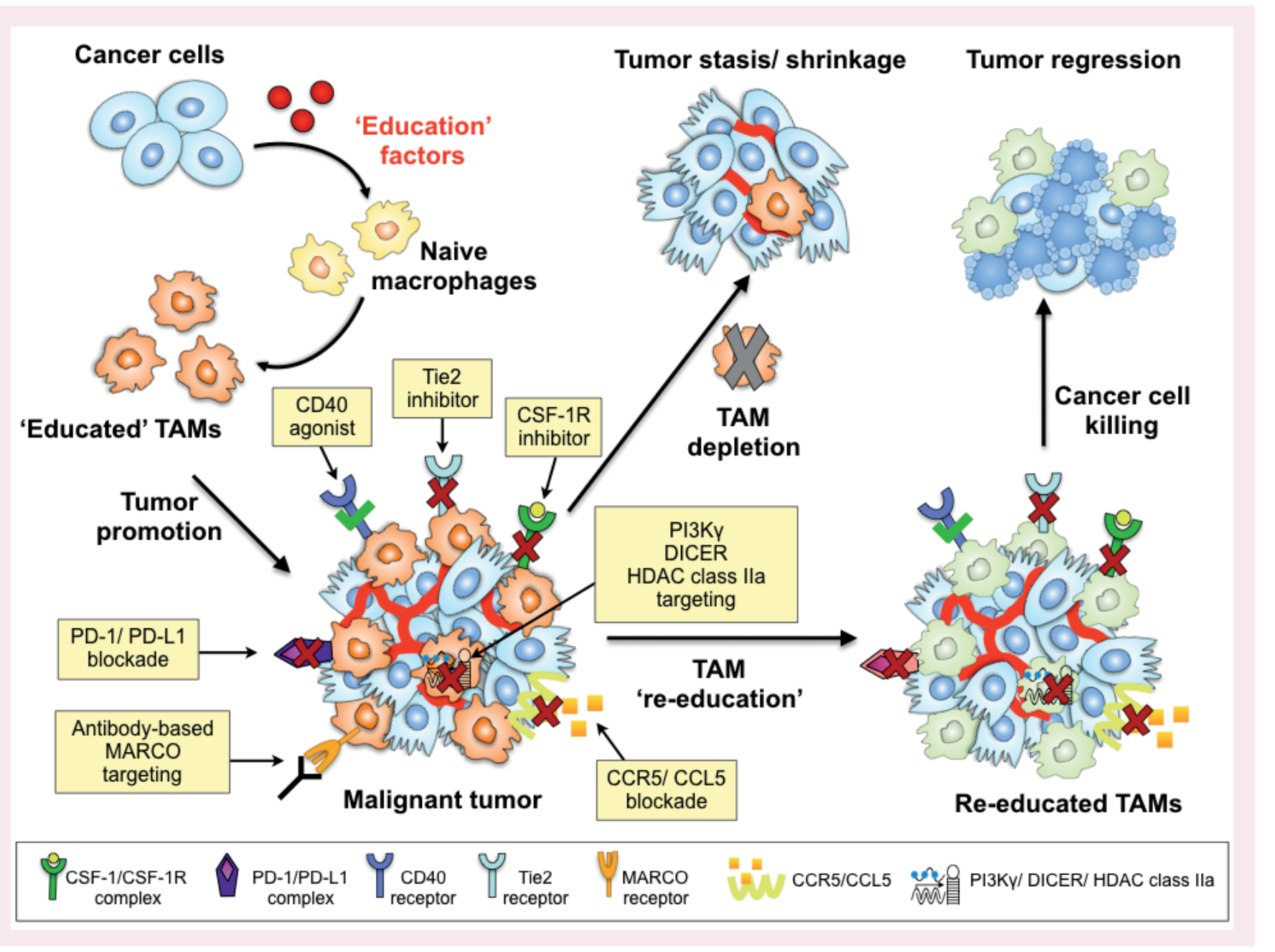

Figure 1. Macrophage targeting strategies in cancer. The plasticity of macrophages and their high capacity to adapt to external signals allow cancer cells to hijack their biological functions. In the majority of solid tumors, macrophages recruited to the tumor niche are educated to acquire protumoral phenotypes. Several strategies have been developed to target macrophages in the tumor microenvironment, including their killing or re-education. Most macrophage-targeted therapies are currently focused on CSF-1R inhibitors, which either deplete or reprogram macrophages. Macrophages can also be targeted via a CD40 agonist that boosts the antitumoral response. Another approach is via disruption of macrophage-derived PD-1 and its interactions with PD-L1 on other cell types. CCL5-CCR5 blockade can result in the re-education of macrophages and subsequent tumor regression. Reprogramming of macrophages can also be achieved by either pharmacologic inhibition or genetic deletion of PI3Kgamma, Tie2, DICER or histone deacetylase class Ila, or via antibody-based targeting of the macrophage-derived protein MARCO.

most numerous and critical cell types across the majority of cancers analyzed, with potent multifaceted roles in regulating cancer progression and modulating the response to therapeutic intervention (Figure 1).

An early clue as to the importance of TAMs in the TME came from analyses of patient samples, in which high numbers of TAMs correlated with poor prognosis for the majority of cancers examined [4]. Several subsequent metaanalyses, with cumulative numbers in the thousands [5,6], have borne out these early findings to confirm that TAM abundance is associated with worse patient prognosis in the vast majority of solid tumors, with colorectal cancer representing one consistent exception to this correlation. However, the simple presence of macrophages per se in the TME does not necessarily indicate a tumor-promoting microenvironment. Instead, it is the precise phenotype of the macrophage, which results from both its ontogeny and tumor-mediated education, that determines its functions and consequently how one would aim to therapeutically target these tumor-associated cells (Table $1 \&$ Table 2).

Macrophages are central guardians of the body that continuously patrol and monitor their surroundings in order to detect perturbations to the homeostatic state, such as infection and cell death. When abnormalities are detected, macrophages quickly adapt to changes in the surrounding microenvironment [7]. Through a wide variety of intra- 
Table 1. Monotherapies used to re-educate tumor-associated macrophages.

\begin{tabular}{|lll|}
\hline Molecular target & Therapeutic agent & Study (year) \\
\hline CSF-1/CSF-1R & Small molecule inhibitor: BLZ945 & Pyonteck et al. (2013); Quail et al. (2016) \\
\hline & Small molecule inhibitor: PLX3397 & Cuccarese et al. (2017); Yan et al. (2017); Zhu et al. (2014) \\
\hline & Small molecule inhibitor: PLX6134 & Perry et al. (2018) \\
\hline CCL5-CCR5 & Anti-CSF-1 antibody & Zhu et al. (2014) \\
\hline CD40 & Anti-CSF-1R antibody & Hoves et al. (2018); Wiehagen et al. (2017) \\
\hline PD-1/PD-L1 & Small molecule inhibitor: Maraviroc & Halama et al. (2016) \\
\hline & Monoclonal agonist antibody & Beatty et al. (2011); Beatty et al. (2013); Hoves et al. \\
\hline & (2018); Perry et al. (2018); Wiehagen et al. (2017) \\
\hline DICER & PD-L1 genetic deletion & Gordon et al. (2017) \\
\hline IL-12 & Anti-PD-1 antibody & \\
\hline HDAC & Anti-human PD-L1 small protein HAC & Baer et al. (2016) \\
\hline PI3K $\gamma$ & Genetic deletion & Saha et al. (2017) \\
\hline & Viral delivery & Guerriero et al. (2017) \\
\hline & TMP195 & Kaneda et al. (2016) \\
\hline MARCO & Small molecule inhibitor: TG100-115 & De Henau et al. (2016); Kaneda et al. (2016) \\
\hline TIE2 & Genetic deletion & Georgoudaki et al. (2016) \\
\hline
\end{tabular}

Table 2. Studies incorporating the re-education of tumor-associated macrophages to enhance the efficacy of other therapeutic interventions.

\begin{tabular}{|c|c|c|}
\hline Molecular target & Combination treatment & Study (year) \\
\hline \multirow[t]{3}{*}{ CSF-1/CSF-1R } & Anti-PD-1, anti-CTLA-4 antibody, gemcitabine & Zhu et al. (2014) \\
\hline & BKM120, FK506, AS1517499, OSI906 & Quail et al. (2016) \\
\hline & Dovitinib, Vatalanib & Yan et al. (2017) \\
\hline PD-1/PD-L1 & Anti-CD47 antibody & Gordon et al. (2017) \\
\hline CD40 & CSF-1/CSF-1R blockade & $\begin{array}{l}\text { Hoves et al. (2018); Perry et al. (2018); Wiehagen et al. } \\
\text { (2017) }\end{array}$ \\
\hline DICER & Anti-CD40, anti-PD-1 antibody & Baer et al. (2016) \\
\hline \multirow[t]{2}{*}{ HDAC } & Carboplatin, paclitaxel & Guerriero et al. (2017) \\
\hline & Anti-PD-1 & \\
\hline \multirow[t]{2}{*}{$\mathrm{PI} 3 \mathrm{~K} \gamma$} & Anti-PD-1 antibody & De Henau et al. (2016); Kaneda et al. (2016) \\
\hline & Anti-CTLA4 antibody & De Henau et al. (2016) \\
\hline MARCO & Anti-CTLA4 antibody & Georgoudaki et al. (2016) \\
\hline TIE2 & Paclitaxel, eribulin & Harney et al. (2017) \\
\hline
\end{tabular}

and extracellular receptors, macrophages respond to a diverse set of stimuli ranging from small molecules such as metabolites, to molecules of intermediate complexity such as cytokines and chemokines, to a family of heterogeneous signaling molecules that are connected with pathogen and danger associated patterns (PAMPs and DAMPs) [8]. Under the influence of these external stimuli, macrophages can oscillate between different activation profiles. Two extreme poles of macrophage activation have been described in detail, originally based on in vitro studies of macrophage biology: pro-inflammatory, classically activated M1 and anti-inflammatory, alternatively activated M2 macrophages. The former produce type I pro-inflammatory cytokines, participate in antigen presentation and have been ascribed antitumorigenic activity, while the latter produce type II cytokines, promote anti-inflammatory responses and have protumorigenic functions $[7,8]$. As these two activation programs of macrophages have opposite effects and have been proposed to be mutually exclusive, their activation status is often referred as 'polarization' toward one or the other extreme.

However, in vivo studies, particularly in the context of cancer, reveal a far more complex landscape than represented by this simple binary classification. The TME is sculpted during its evolution by multiple cell types, 
including cancer cells, which secrete factors that attract and subsequently educate macrophages [9]. The plasticity of macrophages and their high capacity to adapt to external signals allow their biological functions to be hijacked and manipulated in cancer $[9,10]$. Thus, it is now well established that in the majority of solid tumors, macrophages acquire protumoral phenotypes following their recruitment to the tumor niche. Transcriptional profiling of bulk TAMs revealed enrichment in certain M2-like genes, indicating a partial 'alternatively activated' macrophage phenotype [11-14]. Considerable emphasis to date has been placed on defining TAMs in this simple 'either/ or' M1 or M2 state, often to the exclusion of other hypotheses. Indeed, a recent study, taking advantage of single-cell RNA sequencing in breast cancer patient samples, reported expression of both M1 and M2 genes in the same cells, with similar findings reported in gliomas [15,16]. Thus, a comprehensive and integrated approach in which TAMs are rather analyzed based on their ontogeny, TME-mediated education, phenotypic diversity, location within the TME and their resulting tumor-modulating functions, is critical to undertake.

\section{Strategies to deplete or re-educate TAMs}

The high abundance of TAMs within tumors, their genomic stability and rapid response to external stimuli together highlight their potential as a therapeutic target in cancer [17]. The most widely used strategy to date has been via TAM depletion from the tumor mass, through different methods including inhibition of colony stimulating factor-1/colony stimulating factor-1 receptor (CSF-1/CSF-1R) [18,19], CCL2/CCR2 inhibition [20] and delivery of clodronate liposomes [21]. Despite promising results in numerous preclinical animal models of solid tumors, ongoing clinical trials employing the therapeutic strategy of TAM depletion have brought modest or no clinical benefits for cancer patients to date $[17,18,22,23]$. Moreover, cessation of macrophage/ monocyte depletion was shown to actually accelerate tumor growth in a breast cancer metastasis mouse model [24], suggesting possible post-treatment complications and emphasizing the necessity for a much closer evaluation of this approach in preclinical and clinical settings.

Interestingly, several conventional anticancer treatments have been shown to affect macrophage phenotypes [17,25]. Multiple studies have reported the dual nature of the macrophage response to chemotherapy. For example, in both patient-derived xenografts [26] and syngeneic mouse models of breast carcinoma [27,28], paclitaxel treatment induces a recruitment of macrophages to the tumor. In the PyMT-TS1 breast cancer model, TAMs were shown to protect tumor cells from the toxic effects of paclitaxel and other chemotherapies, through non cell-autonomous regulation of mitotic arrest [29] and via the secretion of cathepsin proteases [28]. Newly arrived TAMs, particularly Tie2+ perivascular macrophages, can in turn help tumor cells to disseminate [26] and form metastases. On the other hand, there is also evidence that paclitaxel can repolarize macrophages through TLR4 signaling toward a more pro-inflammatory phenotype [30], which controls the response of melanomas to paclitaxel. Thus, chemotherapy can either induce TAM re-education or enhance their tumor-promoting functions. Similar divergent results were reported for the effects of radiotherapy on TAM biology [17,31]. The effect of irradiation on TAM biology depends on the type, dose and localization of irradiation [31]. Thus, more tailored approaches to interfere with TAM biology may represent a better approach to achieve efficient macrophage re-education, such as antibody-based targeting of the macrophage-derived protein MARCO [32] and other molecularly restricted strategies which are described in detail below.

An antitumoral strategy that has proven successful in mouse glioma models relied on macrophage-tailored reeducation rather than their depletion [33,34]. Administration of two independent, chemically distinct small molecule inhibitors of CSF-1R (BLZ945, PLX3397) resulted in macrophage depletion from healthy tissues including the brain, as expected. Surprisingly, TAMs were not depleted within the glioma microenvironment, but were rather protected from cell death by glioma-supplied cytokines including GM-CSF and IFN- $\gamma$, and persisted in the face of CSF-1R inhibition [33,34]. In-depth analysis of these surviving TAMs revealed changes in their transcription profile with a reprogramming into a less protumoral phenotype in association with significantly augmented phagocytic capacities. This led to rapid tumor regression in a 7-day preclinical trial [33,34], suggesting that TAM-centered re-education might be most efficient as an antitumor therapeutic strategy [1,35]. In addition, the PLX3397-based re-education of TAMs in vivo rendered glioma cells sensitive to different tyrosine kinase inhibitors (TKIs), leading to tumor regression [34]. In combination trials performed for just one week, more than half of the tumors showed $>30 \%$ regression in volume, while in the PLX3397 monotherapy arm approximately 3\% of the tumors reached this level of regression, with most tumors showing a growth stabilization, and none of the tumors treated with the other TKIs alone regressed [34]. 
Here, we discuss recent research that extends this concept beyond gliomas and demonstrates the feasibility of TAM re-education in the TME across diverse cancer types and its beneficial effect on tumor regression and enhancing response to other agents in combination trials (Table $1 \&$ Table 2). The persistence of macrophages in gliomas treated with CSF-1R inhibitors was an unexpected finding [33], nonetheless, similar observations have subsequently been reported for other tumor types. In a pancreatic ductal adenocarcinoma (PDAC) mouse model, therapeutic use of the CSF-1R inhibitor PLX3397 decreased total TAM numbers, yet a subset of macrophages persisted [36]. These remaining TAMs showed a distinct transcriptional profile to PDAC-associated macrophages in steady state, with higher expression of genes involved in antitumor immunity and antigen presentation, among others. Similarly, treatment with a neutralizing antibody against the CSF-1 ligand resulted in a selective decrease in protumoral CD206 ${ }^{+}$TAMs in the PDAC model, thereby skewing the immunosuppressive microenvironment, as CD206- TAMs persisted [36. Thus, in both of these representative examples, while the strategy of CSF-1/ CSF-1R inhibition led to a partial depletion of TAMs, the reprogramming of the subset of TAMs which survived had important effects on the pancreatic TME. Moreover, overall changes in the TAM compartment induced by CSF-1/CSF-1R blockade significantly enhanced the response to immune checkpoint inhibitors (programmed cell death protein 1 [PD-1] and cytotoxic T-lymphocyte-associated protein 4 [CTLA-4] antagonists) combined with chemotherapy [36], revealing the further potential of TAM-targeted therapies when administered in multitargeted approaches. Interestingly, in an implantable mouse model of lung adenocarcinoma deficient for KRAS and p53, PLX3397 treatment resulted in a tumor volume that was approximately tenfold lower when compared with control animals, without decreasing overall TAM density [37]. Instead, the spatial distribution of TAMs was altered within the treated tumors when compared with untreated controls. These results suggest that the lung adenocarcinoma TME, similarly to glioma [33,34], specifically protected TAMs from PLX3397-mediated cell death within the tumor niche [37].

Importantly, recent findings have shown that the efficacy of CSF-1/CSF-1R blockade can be blunted by dynamic adaptive changes within the TME. In gliomas, where macrophages can comprise up to $30 \%$ of total tumor mass [33,38-40], CSF-1R-targeted therapies were proven successful in short-term preclinical trials resulting in substantial tumor regression [33,34]. In long-term preclinical trials, while the initial tumor regression was striking and completely penetrant, with prolonged treatment approximately half of the high-grade tumors ultimately recurred [41]. Interestingly, this acquired resistance was a result of dynamic alterations within the brain TME that adapted over time to overcome CSF-1R targeting. Critically, combining CSF-1R blockade with inhibition of any of the factors identified to mediate the recurrence, including IGF-1, PI3K or NFAT, resulted in circumvention of the resistance mechanisms and glioma eradication. Notably, inhibition of any of these factors as a monotherapy did not induce the regression of gliomas, highlighting the dynamic acquisition of new mechanisms of resistance and consequently the exposure of further vulnerabilities over time in the TME of treated tumors.

Another mechanism of resistance to CSF-1R inhibition, accounting for the modest antitumor efficacy of CSF$1 \mathrm{R}$ blockade in diverse models of melanoma, breast, colon and lung tumors, was shown to be mediated by certain cancer-associated fibroblasts that can express CSF-1R [42]. Upon CSF-1R inhibition, the cancer-associated fibroblasts underwent a switch in cytokine production and recruited granulocytic cells to the tumor [42]. As a consequence, only simultaneous targeting of granulocytic cells and macrophages led to decreased tumor growth. In another preclinical study, CSF-1R inhibition was found to actually promote LLC lung cancer metastasis as a consequence of the loss of myeloid cell-supplied IL-15, which is also a survival factor for NK cells [43]. The indirect depletion of NK cells impaired clearance of LLC cells in the circulation, and specifically increased metastatic seeding - though had no effect on established metastases. By exogenously supplying IL-15 in conjunction with CSF-1R inhibition, the authors could restore NK cells and thereby diminish metastasis [43]. Together, these highlighted examples indicate that macrophage targeting may represent a more effective therapeutic strategy when used in combination with other immune/stromal cell-targeted approaches to overcome adaptive or intrinsic resistance mechanisms.

The CSF-1R inhibitor, PLX3397, has been tested in an escalating dose Phase I clinical trial followed by a Phase II extension study in patients with advanced giant cell synovial tumors that overexpress CSF-1. Results from these studies indicated that PLX3397 was well tolerated and in the extension study, 52\% of patients experienced an antitumor response after treatment. In a Phase II study in patients with recurrent glioblastoma (following the standard of care therapy), treatment with PLX3397 was well tolerated, but did not improve progression-free survival compared with those in the radiotherapy and temozolomide group [44]. Another CSF-1R inhibitor, BLZ945, is 
currently under assessment in a trial in advanced-stage solid tumors either as a single agent or in combination with the anti-PD-1 antibody (NCT02829723).

In addition to CSF-1/CSF-1R inhibitors, a number of other strategies have been found to result in TAM re-education. For example, a CD40 agonist was reported as a potent agent to repolarize macrophages and boost antitumoral immune responses in a PDAC mouse model [45]. Administration of the CD40 agonist induced secretion of the chemokine CCL2 by tumor tissue and from circulating monocytes and resulted in monocyte/macrophage accumulation in the PDAC lesion [46]. This therapeutic intervention also elevated systemic production of IFN$\gamma$, which polarized circulating monocytes prior to their arrival at the tumor site. Importantly, the beneficial effect of this treatment was abolished when CCL2 or Ly6C-expressing monocytes/macrophages were depleted [46]. Thus, in this PDAC model, monocytes were first activated/polarized by therapy-induced systemic changes toward a proinflammatory phenotype, and then subsequently recruited to the tumor site where they drove fibrotic degradation and a decrease in collagen within the TME. The remodeling of extracellular matrix in PDACs led to partial tumor stabilization and sensitization of these tumors to gemcitabine [46]. CD40 agonists are now being explored clinically as a therapeutic approach in diverse solid tumors [47]. For example, the agonistic anti-CD 40 antibody CP-870,893 was tested in an escalating Phase I study and has been well tolerated, resulting in antitumor activity [48]. RO7009789, another CD40 agonist, is currently being investigated in four combinatorial clinical trials in advanced-stage solid tumors (NCT02760797), (NCT02304393), (NCT02665416), (NCT025843).

Interestingly, several studies found that the combination of different CD 40 agonists with CSF-1/CSF-1R pathway inhibition amplified antitumoral effects and resulted in a more potent tumor regression than treatment with single agents [49-51]. In three complementary studies which investigated a broad spectrum of tumors including genetically engineered melanoma [50], implantable solid tumor models of colon adenocarcinoma and carcinoma [49,51], breast adenocarcinoma and sarcoma [49], combination treatments resulted in an amplification of changes in the TAM compartment composition. Immunosuppressive M2-like TAMs were depleted while the remaining TAMs were induced to secrete proinflammatory cytokines, including TNF- $\alpha$, and expressed higher levels of $\mathrm{NOS}_{2}$, thereby rendering tumors susceptible to T-cell-mediated attack. Moreover, CD40-CSF-1R combinatorial targeting had a synergistic effect with anti-PDL1 treatment and led to a complete response in $90 \%$ of tumors in a preclinical study of colon adenocarcinoma [52].

Indeed, a recent study showed that repolarization of TAMs can also be achieved by disruption of macrophagederived PD-1 and external PD-L1 interactions [53]. In colon carcinoma models, PD-1 was expressed not only on T cells, but also on a subset of TAMs that accumulated with tumor progression over time in the TME. These cells were characterized by low MHC II levels, and high expression of CD206 and CD11c, in line with M2-like polarization. PD-1/PD-L1 blockade led to TAM re-education as evidenced by increased phagocytosis. The combined treatment resulted in a significant reduction in tumor growth in both immunocompetent and immunocompromised (NSG and Rag2/Il2r $\gamma^{-/-}$) animals [53]. As the latter lack a functional adaptive immune system, the antitumoral effect of the treatment was largely mediated by macrophages. Indeed, if TAMs were first depleted by a CSF-1R blocking antibody before the combination treatment was initiated, the beneficial effects of PD-L1 neutralization were lost. Of note, the depletion of macrophages also led to a slower tumor growth when compared with untreated controls at the same time points. Finally, when PD-1/PD-L1 blockade was combined with an anti-CD47 antibody, preventing its interaction with SIRP $\alpha$ - which inhibits the recognition and phagocytosis of tumor cells by macrophages, an additive effect of the therapies was observed.

Reprogramming of macrophages has also been shown by either pharmacologic inhibition or genetic deletion of the $\gamma$ isoform of phosphoinositide 3-kinase (PI3Kgamma) [54]. The ablation of PI3K $\gamma$ resulted in suppression of tumor growth in several cancer models, including lung, breast and head and neck squamous cell carcinoma. Detailed analysis revealed that the treatment led to a switch in TAM phenotype with induced expression of MHC II, while the total numbers of TAMs in the TME remained similar to control tumors. Repolarization of TAMs together with a higher abundance of proinflammatory cytokines in the TME resulted in immune system activation and slower tumor growth. Importantly in a lung carcinoma model, both the re-education of macrophages and TAM depletion, either by a CSF-1R-targeting antibody or clodronate liposomes, had a similar inhibitory effect on tumor growth as PI3K $\gamma$ inhibition, but no additive effect of two strategies when combined was observed. In addition, PI3K $\gamma$ inhibition sensitized tumors to immune checkpoint inhibitors [54,55], again showing the importance of macrophage-mediated priming of the TME for optimal immunotherapy efficacy (Table 2).

Another promising strategy for targeting macrophages via Tie2 TKIs was demonstrated during metastatic dissemination. In the 'tumor microenvironment of metastasis (TMEM)' that occurs within the primary tumor in 
close proximity to the blood vessels, Tie $2^{\text {hi }}$ macrophages were shown to accumulate and induce local disruption of vascular junctions, transient vascular permeability, and tumor cell intravasation [56]. In a subsequent study using a MMTV-PyMT orthotopic model, the Tie2 inhibitor rebastinib significantly reduced the growth of implanted mammary carcinomas by $75 \%$, and when combined with paclitaxel resulted in an additional reduction in tumor growth by $90 \%$ [57]. Similarly, rebastinib alone reduced the formation of lung metastasis by $72 \%$, and by $93 \%$ in combination with paclitaxel. Rebastinib also significantly inhibited liver metastasis by $75 \%$ compared with controls in the RIP1-Tag2 pancreatic neuroendocrine tumor model [57]. Examination of the effects of rebastinib at the cellular level demonstrated a reduction in Tie 2 myeloid cell infiltration, anti-angiogenic effects, and blockade of tumor cell intravasation mediated by perivascular Tie $2^{\text {hi }} / \mathrm{VEGFA}^{\text {hi }}$ macrophages in the tumor microenvironment of metastasis [57]. However, in a small Phase I clinical trial of rebastinib as a single agent in leukemia patients, despite the systematic TIE2 inhibition measured by altered levels of Angiopoietin2 (ANG2), this resulted in only a modest clinical benefit (NCT00827138).

The TME is rich in factors that modulate the biology of stromal and immune cells, including macrophages, which enables their education toward a protumoral state. In liver metastases of colorectal cancer patients, one such molecule that was recently described is the chemokine CCL5. Interestingly, this factor was supplied by lymphocytes recruited to the invasive margin of metastatic lesions and promoted tumor growth [58]. When CCL5CCR5 signaling was blocked either by targeting CCR5, which is broadly expressed in the tumor tissue, or via a neutralizing antibody for CCL5, metastatic lesion explants showed signs of necrosis and shrinkage. This effect was entirely mediated by macrophages as clodronate liposome treatment abolished the antitumoral potency of CCL5-CCR5 inhibition. Importantly, the depletion of macrophages alone had no antitumoral effect. Thus, only the re-education of TAMs was capable of inducing tumor cell death, which consequently led to tumor regression in this context. The therapeutic potential of CCL5-CCR5 blockade has been further confirmed in initial results from a clinical trial [58]. In a small cohort of patients with advanced metastatic colorectal cancer, CCL5-CCR5 blockade was well tolerated, and associated with a decrease in tumor-promoting factors, confirming the absence of significant side effects for the CCR5 small molecule inhibitor maraviroc [58].

Molecular mechanisms governing the education of macrophages toward protumoral phenotypes and functions are currently only partially understood. Once the full spectrum of these molecular pathways is revealed, the possibilities for their therapeutic targeting in cancer should inevitably follow. As a representative example, a recent study showed that macrophages deficient for DICER, an enzyme involved in miRNA biogenesis, are resistant to tumor-derived signals and consequently do not undergo polarization to an M2-like phenotype [59]. Macrophagespecific deletion of DICER led to a slower growth of breast, lung and colon implanted solid tumors, where tumor weight was decreased by twofold compared with wild-type control animals at the trial end point. Additionally, the pulmonary metastasis load was decreased in both spontaneous and intravenously injected metastasis models when DICER was deleted in the macrophages. Moreover, the TME populated by DICER-deficient TAMs was characterized by activation of the IFN- $\gamma /$ STAT 1 signaling pathway, which rendered it more proinflammatory with a higher abundance of infiltrating $\mathrm{CD}^{+} \mathrm{T}$ cells. Importantly, the depletion of these DICER-null macrophages via a CSF-1R antibody had detrimental effects as this then accelerated tumor growth in colon and lung carcinomas. Taken together, these results show the beneficial role of TAMs in tumor suppression if they are directed toward a proinflammatory phenotype. Moreover, DICER deficiency in TAMs resulted in better control of tumor growth by the immune system when stimulated either by anti-PD-1 or anti-CD40 treatments, which led to colon carcinoma eradication in 16 of 20 , and 10 of 10 , treated animals respectively at the trial end point [59]. This and other representative examples discussed above demonstrate how TAM re-education can have consequences for the entire TME, rendering tumors more sensitive to immune checkpoint blockade.

Another promising strategy to treat cancer is via engineered oncolytic viruses that are designed to selectively kill cancer cells without affecting the viability of stromal and immune cells within the TME [60]. A recent study explored the therapeutic use of oncolytic viruses encoding IL-12, a proinflammatory cytokine that was previously shown to be important for the antitumoral capabilities of myeloid cells [61]. The delivery of viral particles to glioblastomas resulted in the expected cancer cell death, in addition to the skewing of TAMs toward a more proinflammatory phenotype [62]. This activation of TAMs rendered the gliomas sensitive to anti-PD-1 and anti-CTLA- 4 inhibitors when used together with the virus treatment, resulting in a significant survival benefit and eradication of the gliomas in $89 \%$ of treated animals. Importantly, tumor-bearing animals that were effectively cured with the combination of oncolytic viruses and checkpoint inhibitors were immune to subsequent experimental implantation of glioma 
cells in the contralateral hemisphere [62]. Histological analysis of the brain did not reveal any detectable tumor cells in either hemisphere after 9 months post-treatment. Once again, this example underscores how manipulating the activation status of macrophages can render tumors sensitive to otherwise inefficient therapeutic interventions.

Finally, a recent report demonstrated the role of protein deacetylases in TAM education. Treatment with a small molecule inhibitor of class IIa histone deacetylases, TMP195, resulted in stabilization of the tumor volume [63]. TMP195 treatment also resulted in an approximately threefold lower frequency of pulmonary metastasis in the MMTV-PyMT transgenic model of breast cancer [63]. Detailed gene expression analyses revealed that TMP195 induced a switch in the activation profile of TAMs in mammary tumors, while it had no effect on $\mathrm{CD}^{+}$tumorassociated lymphocytes. This alteration was caused by a simultaneous decrease in the protumoral subset of TAMs defined as CD $11 b^{\text {low }}$ and an increased abundance of CD $11 b^{\text {high }}$, F4/80 and MAC-2 expressing cells. Consequently, the re-educated TAMs showed higher phagocytic capacity and induced normalization of the tumor vasculature. Importantly, simple depletion of all TAM subsets with an anti-CSF-1 antibody did not result in any tumor regression and it abrogated the effects of the TMP195 drug. These results again highlight that re-education of TAMs rather than their depletion can induce more profound changes in the TME that lead to the efficient inhibition of tumor growth and decrease in the metastases occurrence in this example, or bona fide tumor regression in other cancers. Similar to the studies discussed above, when TAMs were re-educated by TMP195, MMTV-PyMT tumors then became susceptible to PD-1/PD-L1 blockade, which was ineffective as monotherapy [63].

\section{Conclusions}

Together, the representative studies discussed in this perspective highlight a growing body of evidence that TAM education not only results in a diverse array of tumor-promoting functions for these cells, but also orchestrates a plethora of immune system-tumor interactions thereby representing a major obstacle for the efficacy of various immunotherapies. As such, the re-education of TAMs to overcome an immunosuppressive environment presents an attractive means of attack, as exemplified by the studies discussed herein. Particularly in cases where TAM depletion deos not deliver a pronounced therapeutic effect, manipulation of the TME via TAM re-education can offer a promising alternative strategy. Although TAM targeting and re-education can represent a potent approach as a monotherapy [17], it may result in the development of resistance and tumor rebound [41], or even accelerate tumor growth once therapy is ceased [24]. On the other hand, the antitumoral potential of TAM-targeted therapies is greatly enhanced when combined with other immune-centered therapies such as targeting the PD-1/PD-L1 and CTLA-4 immune checkpoints. In several preclinical tumor models, this approach was shown to unleash antitumor immunity, leading to tumor regression and even apparent disease eradication. Thus, the potential of TAM-directed therapies lies in combination with other treatments such as immune checkpoint inhibitors or chemotherapy, which is expected to be broadly explored in the coming years.

\section{Future perspective}

Despite the promising results discussed here, particularly in preclinical models, many questions remain unanswered and further studies are needed to deeply understand the complexity of TAM biology. Several studies have shown that TAMs can be phenotypically heterogeneous within a single tumor $[36,49,53]$, and recent reports have revealed that ontologically distinct cells can be both similarly and differentially educated within a single TME. For example, in brain malignancies [38,64], lung cancer [65], and in PDAC models [66], both tissue-resident and bone marrowderived macrophages are recruited to the developing tumor mass and further educated in situ by cancer cells, adding multiple layers of complexity. With these new findings revealing distinct TAM progenitor pools, and different modes of tumor-mediated education, several important and intriguing questions are raised: Does ontogeny imprint the function of TAMs in the TME and also in response to various therapies? Should we move from general TAM targeting to therapies directed to TAM subpopulations? If so, which subset of TAMs is more prone to be re-educated, and which represents the most potent therapeutic target? Will TAM re-education strategies need to be adapted for individual organs and TMEs? Another general challenge is to translate promising preclinical results into the clinic. Macrophage-centered therapies have so far been evaluated mostly as monotherapies, with multiple preclinical studies demonstrating the feasibility and efficacy of this approach. However, TAM-directed monotherapies have provided limited benefit for patient health in the clinical trials reported to date [25]. Thus, clinical trials evaluating TAM-focused therapies in rational combinations with other immunotherapeutic interventions, or other anticancer agents, are clearly needed going forward to achieve beneficial outcomes for cancer patients. Addressing these 
Tumor-associated macrophages in the cancer microenvironment

- Tumors develop and progress within a complex tissue environment that collectively comprises the tumor microenvironment (TME).

- Tumor-associated macrophages (TAMs) represent one of the most abundant nontransformed cell types present within the TME.

- Macrophages can acquire protumoral phenotypes during the process of their education within the TME, and their abundance and tumor-promoting phenotypes correlate with poor patient prognosis.

- Multiple strategies are being developed to target TAMs as anticancer therapies.

Strategies to deplete or re-educate TAMs

Targeting of macrophages via the CSF-1/CSF-1R pathway

- CSF-1/CSF-1R inhibition leads either to depletion or re-education of TAMs within the TME and has antitumoral effects in several preclinical cancer models.

- CSF-1/CSF-1R inhibition can be circumvented by acquired changes in the TME. However, this can be overcome by combining CSF-1R blockade with targeting of other TME-derived protumorigenic factors.

Targeting of macrophages via CD40

- CD40 agonists repolarize macrophages and boost antitumoral immune responses.

- Combination of different CD40 agonists with CSF-1/CSF-1R pathway inhibition amplifies antitumorigenic effects and results in a more potent tumor regression compared with single agents across a large panel of preclinical cancer models.

- Combination of anti-CD40 and anti-CSF-1R positively enhances the effect of anti-PD-L1 treatment.

Targeting of macrophages via PD-1 inhibition

- The therapeutic effect of PD-1/PD-L1 targeting can be mediated by PD-1+ macrophages in both immunocompetent and immunocompromised animals.

- The beneficial effect of PD-1 can be abrogated in the absence of tumor-associated macrophages.

Targeting of macrophages via kinase inhibition

- Ablation of macrophage-supplied PI3K $\gamma$ in tumor-bearing animals suppresses tumor growth.

- $\mathrm{PI} 3 \mathrm{~K} \gamma$ inhibition results in TAM repolarization and immune system activation that together delay tumor growth.

- $\mathrm{PI3K} \gamma$ inhibition sensitizes tumors to immune checkpoint inhibitors.

- Tie2 is expressed by a subset of tumor-associated macrophages involved in metastatic dissemination.

- Tie2 inhibition decreases metastatic burden.

Targeting of macrophages via CCL5/CCR5 pathway

- Blockade of CCL5-CCR5 signaling results in macrophage activation and necrosis of metastatic lesions in a preclinical model.

- A subsequent clinical trial supported the therapeutic potential of CCL5-CCR5 targeting.

Targeting of macrophages via DICER pathway

- DICER deletion in macrophages results in slower tumor growth and lower incidence of metastases in cancer models.

- Depletion of DICER-deficient TAMs specifically via an anti-CSF-1R antibody accelerates tumor growth.

- DICER-deficient TAMs render the TME more proinflammatory, and enables the beneficial effect of both anti-PD-1 and anti-CD40 treatments.

Targeting of macrophages via oncolytic viruses

- Delivery of oncolytic viruses encoding IL-12 results in cancer cell death and skews TAMs toward a proinflammatory phenotype.

- Viral treatment combined with anti-PD-1 and anti-CTLA-4 inhibitors leads to eradication of preclinical gliomas in the majority of treated animals.

Targeting of macrophages via class lla histone deacetylase

- Treatment with the class Ila histone deacetylase inhibitor TMP195 results in stabilization of tumor volume and a reduced frequency of spontaneous metastases in preclinical trials.

- TMP195 acts on TAMs and induces a higher phagocytic capacity and normalization of the tumor vasculature.

- TMP195 unmasks an antitumor effect of PD-1/PD-L1 blockade in otherwise insensitive MMTV-PyMT tumors.

Conclusions

- Therapeutic targeting of macrophages is a feasible approach that can lead to antitumorigenic effects.

- TAM re-education renders tumors susceptible to otherwise inefficient immunotherapies, thus TAMs emerge as a key orchestrator of the immune response against cancer.

different challenges should then facilitate the tailoring of TAM-targeted therapies in order to induce prolonged antitumor efficacy across diverse cancer types. 
Financial \& competing interests disclosure

Research in the Joyce lab is currently supported by the Swiss Cancer League, Swiss Bridge Award, Breast Cancer Research Foundation, Cancer Research UK, Ludwig Institute for Cancer Research, and the University of Lausanne. The authors have no other relevant affiliations or financial involvement with any organization or entity with a financial interest in or financial conflict with the subject matter or materials discussed in the manuscript apart from those disclosed.

No writing assistance was utilized in the production of this manuscript.

\section{Acknowledgments}

We thank all members of the Joyce lab for general discussions and insights into the topic of macrophage re-education in cancer.

Author contributions

J Kowal, M Kornete and J Joyce have designed and written the article.

\section{Open access}

This work is licensed under the Attribution-NonCommercial-NoDerivatives 4.0 Unported License. To view a copy of this license, visit http://creativecommons.org/licenses/by-nc-nd/4.0/

\section{References}

Papers of special note have been highlighted as: $\bullet$ of interest; $\bullet \bullet$ of considerable interest

1. Quail DF, Joyce JA. Microenvironmental regulation of tumor progression and metastasis. Nat. Med. 19(11), 1423-1437 (2013).

2. Hanahan D, Weinberg RA. Hallmarks of cancer: the next generation. Cell 144(5), 646-674 (2011).

3. Egeblad M, Nakasone ES, Werb Z. Tumors as organs: complex tissues that interface with the entire organism. Dev. Cell 18(6), 884-901 (2010).

4. Bingle L, Brown NJ, Lewis CE. The role of tumour-associated macrophages in tumour progression: implications for new anticancer therapies. J. Pathol. 196(3), 254-265 (2002).

5. Fridman WH, Zitvogel L, Sautes-Fridman C, Kroemer G. The immune contexture in cancer prognosis and treatment. Nat. Rev. Clin. Oncol. 14(12), 717-734 (2017).

6. Zhang QW, Liu L, Gong CY et al. Prognostic significance of tumor-associated macrophages in solid tumor: a meta-analysis of the literature. PLoS ONE 7(12), e50946 (2012).

7. Wynn TA, Chawla A, Pollard JW. Macrophage biology in development, homeostasis and disease. Nature 496(7446), 445-455 (2013).

8. Glass CK, Natoli G. Molecular control of activation and priming in macrophages. Nat. Immunol. 17(1), 26-33 (2016).

9. Noy R, Pollard JW. Tumor-associated macrophages: from mechanisms to therapy. Immunity 41(1), 49-61 (2014).

10. Pollard JW. Tumour-educated macrophages promote tumour progression and metastasis. Nat. Rev. Cancer 4(1), 71-78 (2004).

11. Colegio OR, Chu NQ, Szabo AL et al. Functional polarization of tumour-associated macrophages by tumour-derived lactic acid. Nature 513(7519), 559-563 (2014)

12. Ojalvo LS, King W, Cox D, Pollard JW. High-density gene expression analysis of tumor-associated macrophages from mouse mammary tumors. Am. J. Pathol. 174(3), 1048-1064 (2009).

13. Pucci F, Venneri MA, Biziato D et al. A distinguishing gene signature shared by tumor-infiltrating Tie2-expressing monocytes, blood 'resident' monocytes, and embryonic macrophages suggests common functions and developmental relationships. Blood 114(4), 901-914 (2009).

14. Biswas SK, Gangi L, Paul S et al. A distinct and unique transcriptional program expressed by tumor-associated macrophages (defective NF-kappaB and enhanced IRF-3/STAT1 activation). Blood 107(5), 2112-2122 (2006).

15. Azizi E, Carr AJ, Plitas G et al. Single-cell map of diverse immune phenotypes in the breast tumor microenvironment. Cell 174 (5), 1293-1308 (2018).

-. Taking advantage of paired human biopsies, this study characterized tumor-infiltrating immune cells by means of single-cell RNA-seq, highlighting tumor-specific states of activation of several immune components of the breast tumor microenvironment (TME).

16. Muller S, Kohanbash G, Liu SJ et al. Single-cell profiling of human gliomas reveals macrophage ontogeny as a basis for regional differences in macrophage activation in the tumor microenvironment. Genome Biol. 18(1), 234 (2017).

17. Mantovani A, Marchesi F, Malesci A, Laghi L, Allavena P. Tumour-associated macrophages as treatment targets in oncology. Nat. Rev. Clin. Oncol. 14(7), 399-416 (2017).

18. Majety M, Runza V, Lehmann C, Hoves S, Ries CH. A drug development perspective on targeting tumor-associated myeloid cells. FEBS J. 285(4), 763-776 (2018). 
- CD40-dependent depolarization of macrophages in the TME was found to boost anti-tumoral immune responses, and further enhanced by concomitant inhibition of the CSF-1/CSF-1R pathway. This indicates that macrophage-based targeting can be tailored and amplified to increase therapeutic efficiency.

19. Ries $\mathrm{CH}$, Cannarile MA, Hoves $\mathrm{S}$ et al. Targeting tumor-associated macrophages with anti-CSF-1R antibody reveals a strategy for cancer therapy. Cancer Cell 25(6), 846-859 (2014).

20. Qian BZ, Li J, Zhang H et al. CCL2 recruits inflammatory monocytes to facilitate breast-tumour metastasis. Nature 475(7355), 222-225 (2011).

21. Van Rooijen N, Hendrikx E. Liposomes for specific depletion of macrophages from organs and tissues. Methods Mol. Biol. 605, 189-203 (2010).

22. Peyraud F, Cousin S, Italiano A. CSF-1R inhibitor development: current clinical status. Curr. Oncol. Rep. 19(11), 70 (2017).

23. Cannarile MA, Weisser M, Jacob W, Jegg AM, Ries CH, Ruttinger D. Colony-stimulating factor 1 receptor (CSF1R) inhibitors in cancer therapy. J. Immunother. Cancer 5(1), 53 (2017).

24. Bonapace L, Coissieux MM, Wyckoff J et al. Cessation of CCL2 inhibition accelerates breast cancer metastasis by promoting angiogenesis. Nature 515(7525), 130-133 (2014).

25. Cassetta L, Pollard JW. Targeting macrophages: therapeutic approaches in cancer. Nat. Rev. Drug Discov. 17, 887-904 (2018).

26. Karagiannis GS, Pastoriza JM, Wang Y et al. Neoadjuvant chemotherapy induces breast cancer metastasis through a TMEM-mediated mechanism. Sci. Transl. Med. 9(397), eaan0026 (2017).

27. Denardo DG, Brennan DJ, Rexhepaj E et al. Leukocyte complexity predicts breast cancer survival and functionally regulates response to chemotherapy. Cancer Discov. 1(1), 54-67 (2011).

28. Shree T, Olson OC, Elie BT et al. Macrophages and cathepsin proteases blunt chemotherapeutic response in breast cancer. Genes Dev. 25(23), 2465-2479 (2011).

29. Olson OC, Kim H, Quail DF, Foley EA, Joyce JA. Tumor-associated macrophages suppress the cytotoxic activity of antimitotic agents. Cell Rep. 19(1), 101-113 (2017).

30. Wanderley CW, Colon DF, Luiz JPM et al. Paclitaxel reduces tumor growth by reprogramming tumor-associated macrophages to an M1 profile in a TLR4-dependent manner. Cancer Res. 78(20), 5891-5900 (2018).

31. Genard G, Lucas S, Michiels C. Reprogramming of tumor-associated macrophages with anticancer therapies: radiotherapy versus chemo- and immunotherapies. Front. Immunol. 8, 828 (2017).

32. Georgoudaki AM, Prokopec KE, Boura VF et al. Reprogramming tumor-associated macrophages by antibody targeting inhibits cancer progression and metastasis. Cell Rep. 15(9), 2000-2011 (2016).

33. Pyonteck SM, Akkari L, Schuhmacher AJ et al. CSF-1R inhibition alters macrophage polarization and blocks glioma progression. Nat. Med. 19(10), 1264-1272 (2013).

-. This study was one of the first to show that tumor-associated macrophage (TAM) re-education, in this case via a CSF-1R inhibitor, can have a potent effect on tumor regression. The authors found that TAM survival factors are specifically produced by gliomas, which leads to TAM reprogramming rather than cell death; in contrast to microglia in the normal brain, which were effectively depleted by CSF-1R inhibition.

34. Yan D, Kowal J, Akkari L et al. Inhibition of colony stimulating factor-1 receptor abrogates microenvironment-mediated therapeutic resistance in gliomas. Oncogene 36(43), 6049-6058 (2017).

35. Bowman RL, Joyce JA. Therapeutic targeting of tumor-associated macrophages and microglia in glioblastoma. Immunotherapy 6(6), 663-666 (2014)

36. Zhu Y, Knolhoff BL, Meyer MA et al. CSF1/CSF1R blockade reprograms tumor-infiltrating macrophages and improves response to T-cell checkpoint immunotherapy in pancreatic cancer models. Cancer Res. 74(18), 5057-5069 (2014).

37. Cuccarese MF, Dubach JM, Pfirschke C et al. Heterogeneity of macrophage infiltration and therapeutic response in lung carcinoma revealed by 3D organ imaging. Nat. Commun. 8, 14293 (2017).

38. Bowman RL, Klemm F, Akkari L et al. Macrophage ontogeny underlies differences in tumor-specific education in brain malignancies. Cell Rep. 17(9), 2445-2459 (2016).

39. Komohara $\mathrm{Y}$, Ohnishi K, Kuratsu J, Takeya M. Possible involvement of the M2 anti-inflammatory macrophage phenotype in growth of human gliomas. J. Pathol. 216(1), 15-24 (2008).

40. Hussain SF, Yang D, Suki D, Aldape K, Grimm E, Heimberger AB. The role of human glioma-infiltrating microglia/macrophages in mediating antitumor immune responses. Neuro Oncol. 8(3), 261-279 (2006).

41. Quail DF, Bowman RL, Akkari L et al. The tumor microenvironment underlies acquired resistance to CSF-1R inhibition in gliomas. Science 352(6288), aad3018 (2016).

-. The authors show that long-term CSF-1R inhibition in different preclinical glioma models can lead to the development of acquired resistance in a subset of animals. The resistance mechanism was found to be independent of tumor cell-intrinsic genetic alterations, but rather mediated by a dynamically changing TME. Consequently, targeting different signaling nodes in the 
paracrine signaling network identified to mediate adaptive resistance, along with CSF-1R inhibition, resulting in sustained glioma regression in the vast majority of mice.

42. Kumar V, Donthireddy L, Marvel D et al. Cancer-associated fibroblasts neutralize the anti-tumor effect of CSF1 receptor blockade by inducing PMN-MDSC infiltration of tumors. Cancer Cell 32(5), 654-668 e655 (2017).

43. Beffinger M, Tallon De Lara P, Tugues $S$ et al. CSF1R-dependent myeloid cells are required for NK-mediated control of metastasis. JCI Insight 17;3(10), pii: 97792 (2018). doi:10.1172/jci.insight.97792

44. Butowski N, Colman H, De Groot JF et al. Orally administered colony stimulating factor 1 receptor inhibitor PLX3397 in recurrent glioblastoma: an Ivy Foundation Early Phase Clinical Trials Consortium Phase II study. Neuro Oncol. 18(4), 557-564 (2016).

45. Beatty GL, Chiorean EG, Fishman MP et al. CD40 agonists alter tumor stroma and show efficacy against pancreatic carcinoma in mice and humans. Science 331(6024), 1612-1616 (2011).

46. Long KB, Gladney WL, Tooker GM, Graham K, Fraietta JA, Beatty GL. IFNgamma and CCL2 cooperate to redirect tumor-infiltrating monocytes to degrade fibrosis and enhance chemotherapy efficacy in pancreatic carcinoma. Cancer Discov. 6(4), 400-413 (2016).

47. Beatty GL, Li Y, Long KB. Cancer immunotherapy: activating innate and adaptive immunity through CD40 agonists. Expert Rev. Anticancer Ther. 17(2), 175-186 (2017).

48. Vonderheide RH, Flaherty KT, Khalil M et al. Clinical activity and immune modulation in cancer patients treated with CP-870,893, a novel CD40 agonist monoclonal antibody. J. Clin. Oncol. 25(7), 876-883 (2007).

49. Hoves $\mathrm{S}$, Ooi $\mathrm{CH}$, Wolter $\mathrm{C}$ et al. Rapid activation of tumor-associated macrophages boosts preexisting tumor immunity. J. Exp. Med. 215(3), 859-876 (2018).

50. Perry CJ, Munoz-Rojas AR, Meeth KM et al. Myeloid-targeted immunotherapies act in synergy to induce inflammation and antitumor immunity. J. Exp. Med. 215(3), 877-893 (2018).

51. Wiehagen KR, Girgis NM, Yamada DH et al. Combination of CD40 agonism and CSF-1R blockade reconditions tumor-associated macrophages and drives potent antitumor immunity. Cancer Immunol. Res. 5(12), 1109-1121 (2017).

52. Xiong H, Mittman S, Rodriguez R et al. Anti-PD-L1 treatment results in functional remodeling of the macrophage compartment. Cancer Res. doi:10.1158/0008-5472.CAN-18-3208 (2019) (Epub ahead of print).

53. Gordon SR, Maute RL, Dulken BW et al. PD-1 expression by tumour-associated macrophages inhibits phagocytosis and tumour immunity. Nature 545(7655), 495-499 (2017).

-• Showed that PD-1 expression is not restricted to $\mathrm{T}$ cells and is also found on macrophages in the TME, thus implying that the efficacy (or not) of PD-1/PD-L1 therapies can be mediated through actions on multiple cell types.

54. Kaneda MM, Messer KS, Ralainirina N et al. PI3Kgamma is a molecular switch that controls immune suppression. Nature 539(7629), 437-442 (2016).

- PI3Kgamma identified as a potent regulator of the macrophage polarization and response to external stimuli. The feasibility of PI3Kgamma targeting was shown using small molecule inhibitors.

55. De Henau O, Rausch M, Winkler D et al. Overcoming resistance to checkpoint blockade therapy by targeting PI3Kgamma in myeloid cells. Nature 539(7629), 443-447 (2016).

56. Harney AS, Arwert EN, Entenberg D et al. Real-time imaging reveals local, transient vascular permeability, and tumor cell intravasation stimulated by TIE2hi macrophage-derived VEGFA. Cancer Discov. 5(9), 932-943 (2015).

57. Harney AS, Karagiannis GS, Pignatelli J et al. The selective Tie2 inhibitor rebastinib blocks recruitment and function of Tie2(Hi) macrophages in breast cancer and pancreatic neuroendocrine tumors. Mol. Cancer Ther. 16(11), 2486-2501 (2017).

58. Halama N, Zoernig I, Berthel A et al. Tumoral immune cell exploitation in colorectal cancer metastases can be targeted effectively by anti-CCR5 therapy in cancer patients. Cancer Cell 29(4), 587-601 (2016).

- Describes a new education factor for macrophages, CCL5, that is provided by lymphocytes in the TME and not by tumor cells directly, thereby shedding light on a potential mechanism by which the whole TME can shape TAM education and not only tumor cells.

59. Baer C, Squadrito ML, Laoui D et al. Suppression of microRNA activity amplifies IFN-gamma-induced macrophage activation and promotes anti-tumour immunity. Nat. Cell Biol. 18(7), 790-802 (2016).

- The authors revealed a role for DICER in macrophage homeostasis and response to external stimuli, such as within the TME. DICER-deficient macrophages were shown to have different activation status and immunoregulatory capacities through which they can alter tumor growth, highlighting the importance of the TME in shaping the dynamics of tumor progression.

60. Kaufman HL, Kohlhapp FJ, Zloza A. Oncolytic viruses: a new class of immunotherapy drugs. Nat. Rev. Drug Discov. 14(9), 642-662 (2015).

61. Kerkar SP, Goldszmid RS, Muranski P et al. IL-12 triggers a programmatic change in dysfunctional myeloid-derived cells within mouse tumors. J. Clin. Invest. 121(12), 4746-4757 (2011).

62. Saha D, Martuza RL, Rabkin SD. Macrophage polarization contributes to glioblastoma eradication by combination immunovirotherapy and immune checkpoint blockade. Cancer Cell 32(2), 253-267 e255 (2017). 
63. Guerriero JL, Sotayo A, Ponichtera HE et al. Class IIa HDAC inhibition reduces breast tumours and metastases through anti-tumour macrophages. Nature 543(7645), 428-432 (2017).

64. Chen Z, Feng X, Herting CJ et al. Cellular and molecular identity of tumor-associated macrophages in glioblastoma. Cancer Res. 77(9), 2266-2278 (2017).

65. Loyher PL, Hamon P, Laviron M et al. Macrophages of distinct origins contribute to tumor development in the lung. J. Exp. Med. 215(10), 2536-2553 (2018)

66. Zhu Y, Herndon JM, Sojka DK et al. Tissue-resident macrophages in pancreatic ductal adenocarcinoma originate from embryonic hematopoiesis and promote tumor progression. Immunity 47(3), 597 (2017). 